\title{
e-Phaïstos
}

e-Phaïstos

Revue d'histoire des techniques / Journal of the history

of technology

V-2 | 2016

Histoire des techniques en Afrique de l'Ouest

\section{Histoire des techniques en Afrique : une introduction}

History of Technology in Africa : an Introduction

\section{François Wassouni}

\section{(2) OpenEdition}

Journals

Édition électronique

URL : http://journals.openedition.org/ephaistos/5710

DOI : 10.4000/ephaistos.5710

ISSN : 2552-0741

Éditeur

IHMC - Institut d'histoire moderne et contemporaine (UMR 8066)

Édition imprimée

Date de publication : 15 juin 2016

Pagination : 8-13

ISSN : 2262-7340

Référence électronique

François Wassouni, «Histoire des techniques en Afrique : une introduction », e-Phaïstos [En ligne], V-2 |

2016, mis en ligne le 15 mars 2020, consulté le 18 juin 2020. URL : http://journals.openedition.org/ ephaistos/5710 ; DOI : https://doi.org/10.4000/ephaistos.5710 


\title{
Introduction
}

\author{
François Wassouni ${ }^{1}$ \\ Université de Maroua \\ Département d'histoire
}

Je voudrais introduire ce dossier d'e-Phaïstos consacré à l'Afrique en marquant un arrêt sur un numéro de la revue Tiers Monde paru en 20132, portant spécialement sur "l'écriture de l'histoire dans les pays en développement ». Les articles réunis dans ce document sont d'une extrême diversité et couvrent plusieurs aires géographiques : Chine, Brésil, Liban et l'Afrique. Pour ce qui concerne le continent africain, les textes de Catherine CoqueryVidrovitch3 et de Robert Ageneau4 captivent de par leur originalité. C'est davantage l'article de la première auteure qui retient l'attention ici, dans la mesure où il étudie l'historiographie africaine en Afrique. L'auteure relève la dynamique de la production historienne par les Africains, laquelle production est dense, riche et variée, qu'il s'agisse de l'Afrique francophone, anglophone, lusophone ou arabophone. Malgré les difficultés qui entourent la pratique de l'activité de recherche, les Africains se sont réapproprié l'écriture de leur histoire et des travaux de qualité sont produits, même si, parfois, ceux-ci ne sont pas toujours diffusés hors du continent5. S'il est évident que l'écriture de l'histoire connaît une vivacité dans la plupart des pays, il n'en demeure pas moins vrai que du point de vue des territoires de l'histoire, beaucoup reste encore à faire. Contrairement aux pays occidentaux où tous les différents champs de l'histoire sont suffisamment investis, en Afrique ce sont surtout des domaines comme la politique, l'économie et la culture qui dominent encore l'historiographie. Et justement, au rang des champs peu investis, figure en très bonne place l'histoire des techniques.
Alors que foisonnent en Occident des laboratoires, des réseaux de recherche, des revues scientifiques $^{6}$ et des institutions de recherche et des créneaux de formation 7 dans cette branche que les Anglo-saxons dénomment par « History of Technology », en Afrique ce n'est véritablement pas encore le cas. Une investigation dans l'historiographie africaine montre que si les techniques ont été étudiées dans bien des pays, ce sont pour la plupart les archéologues qui s'en sont occupés ${ }^{8}$, dans le cadre des travaux sur la culture matérielle des civilisations disparues. Pourtant, ce ne sont ni les sources et encore moins les activités techniques et les différentes problématiques qui les entourent qui font défaut 9 . Dans un contexte de mondialisation où les techniques (modernes surtout) «gouvernent » dorénavant le monde, il est important qu'un intérêt puisse être accordé à cette branche, d'autant que le continent africain accuse un grand retard en la matière. Or, si le continent fait piètre figure en matière de production et de vulgarisation des techniques modernes, il est pourtant riche en éléments relatifs aux techniques anciennes qui constituent un patrimoine historique important et impressionnant (techniques architecturales, artisanales, culinaires, thérapeutiques, d'agriculture, de décoration, vestimentaires, etc). Une meilleure connaissance de la "History of technology » en Afrique ne contribuerait-elle pas à exorciser, tout au moins en partie, les réminiscences des démons du racisme anti-noir, qui s'assoit surtout sur la croyance que l'Afrique n'aurait presque rien apporté au monde sur le plan technologique ${ }^{10}$ ? 
Or, les techniques ont occupé, de tous temps, une place importante dans les sociétés humaines, d'où la difficulté de saisir l'évolution de celles-ci sans prendre en compte les techniques diverses qu'elles avaient et continuent encore d'élaborer. Dans l'histoire, les techniques ont toujours été des éléments fondamentaux dans la vie des hommes. Elles jouent un rôle essentiel, facilitent la vie, résolvent nombre de problèmes auxquels les hommes sont confrontés. Il n'est pas possible de se déplacer, de faire de l'agriculture, de communiquer, de se soigner, de s'habiller, de s'abriter, pour ne citer que ces domaines-là, sans se référer aux techniques. S'il devient de plus en plus facile à travers le monde de communiquer, avec la possibilité de savoir en un temps record ce qui se passe d'un coin à un autre, c'est surtout le fait des techniques. Depuis des millénaires, l'Homme a développé des techniques qui lui ont permis de résoudre ses besoins les plus fondamentaux, lui permettant de transformer les matériaux de la nature à des fins alimentaires, vestimentaires ou militaires. L'écriture d'une histoire pertinente de l'humanité suppose donc forcément la prise en compte de ses savoirs techniques. Dans un ouvrage dirigé par François Dortier, on peut lire ce qui suit à propose des techniques : " Toute l'histoire de l'humanité peut être lue à travers l'histoire des techniques »11. Pour Anne-Françoise Garçon, "l'histoire des techniques est l'histoire des efforts accomplis par l'homme pour contrôler le cadre matériel de son existence afin de d'assurer sa survie d'abord, d'accroître son effort et sa puissance d'action ensuite. [...] L'histoire des techniques apparaît comme un élément capital de l'histoire des sociétés, et participe à la grande interrogation sur le destin de l'humanité à l'aube du XXIe siècle " ${ }^{12}$. On comprend pourquoi dans les années 1930, des historiens comme Marc Bloch et Lucien Febvre avaient vivement plaidé pour que l'histoire prenne en compte les techniques, quoiqu'il fallût attendre les années 1970 pour voir cette branche de l'histoire émerger avec des historiens comme Maurice Daumas $^{13}$, François Russo ou Roland Mousnier ${ }^{14}$.
Le patrimoine technique africain nécessite d'être scruté par les chercheurs de diverses disciplines, les historiens au premier chef, dans le but d'en faire un inventaire, d'étudier la dynamique et les fonctionnalités de ces corps de savoirs à travers les temps ${ }^{15}$. En 2013, l'Université de Paris I Panthéon-Sorbonne rassemblait des textes de jeunes chercheurs pour en faire un excellent petit ouvrage sur les patrimoines techniques et les patrimoines de l'industrie en Afrique $^{16}$. Ces textes de jeunes chercheurs du Master TPTI, leurs enseignants et quelques grands noms de l'archéologie ${ }^{17}$ et du patrimoine technique africains, démontrent la richesse et la diversité de ce patrimoine qui constitue un vaste chantier pour la recherche.

Ce dossier, qui réunit les articles de chercheurs confirmés et de jeunes chercheurs, aborde d'intéressantes problématiques de l'histoire des techniques et permet ainsi de mettre en exergue divers pans de l'histoire des sociétés étudiées. L'un des objectifs visés par cette parution est justement d'interpeler les historiens africains sur l'urgence et la nécessité d'accorder un intérêt à l'histoire des techniques, de l'investiguer, de la documenter et de la développer, pour que prenne également corps une école africaine en la matière. La richesse et la diversité des thèmes étudiés (techniques architecturales, militaires, halieutiques, de la peausserie ou de la vannerie, etc.) montrent qu'il existe de nombreuses sources susceptibles d'aider les historiens à investiguer sans grandes difficultés plusieurs contours de l'évolution des techniques et leurs enjeux, dans les sociétés d'hier et d'aujourd'hui.

Ce dossier fait la part belle aux techniques architecturales avec quatre articles à ce propos : ceux de Mahamane Addo, de Jean-Marie Datouang Djoussou, de Rémy Dzou Tsanga et de Jean Gormo. Les quatre autres textes portent sur les techniques d'élaboration et d'usage du cuir (François Wassouni), les techniques défensives (Noël Lavallière Betga Djenkwé), de pêche (Mahamat Abba Ousman) et de la vannerie (Cyrille Zeh). 
L'article de Mahamane Addo ${ }^{18}$ qui ouvre ce dossier, porte sur la rencontre entre le patrimoine immatériel et matériel dans l'architecture du Soudan Central. Les monuments que présente cet article, témoignent de l'existence d'un important patrimoine immobilier qui traverse plusieurs siècles (XIIIe-XXI ${ }^{\mathrm{e}}$ siècles). Ce patrimoine n'est pas seulement matériel dans la mesure où il incarne aussi un patrimoine immatériel de la zone sahélosoudanienne. Ils constituaient et constituent encore des lieux de mémoire, d'étude et de formation religieuse, politique et sociale. Ce patrimoine où le matériel, le spirituel, le politique et le technique sont intimement liés, témoigne du niveau de technicité atteint par les habitants de la sous-région pendant la période précoloniale.

Partant de l'hypothèse selon laquelle, en Afrique subsaharienne, l'architecture de pierre sèche n'est plus seulement l'apanage de la région comprise entre le Limpopo et le Zambèze, où le référentiel est le Great Zimbabwe, la réflexion de Jean-Marie Datouang Djoussou montre que l'essor de cette technique architecturale dans les monts Mandara septentrionaux camerounais est concomitante à l'apogée du Great Zimbabwe. Pour cet auteur, les résultats des travaux archéologiques effectués sur trois des seize ruines des monts Mandara, révèlent des bornes chronologiques inférieures cadrant avec la période pendant laquelle l'impressionnante architecture de l'Afrique Australe semble le style architectural au travers duquel s'exprime la grandeur des autorités politiques et religieuses locales.

Le travail de Rémy Dzou Tsanga, qui part du postulat selon lequel le Cameroun présente une extraordinaire diversité des formes architecturales, rend compte des particularismes techniques des œuvres architecturales perçues comme des marqueurs d'identité culturelle. Tributaires de la contrainte des milieux écologiques, ces formes démontraient à plus d'un titre l'ingéniosité des sociétés de l'époque à exploiter les matériaux que leur offre la nature pour mettre en place divers procédés de construction. Le contact avec la civilisation occiden- tale a fortement entamé ces savoirs techniques architecturaux qui risquent de s'évanouir dans l'oubli. D'où l'intérêt de ce travail qui pourrait pousser les décideurs à envisager la conservation du patrimoine architectural, lequel reste un facteur important pour le développement du tourisme culturel.

L'article de Jean Gormo ${ }^{19}$, quant à lui, montre le rôle polyvalent que l'arbre a toujours joué dans la vie des sociétés rurales au Nord-Cameroun en général, et celle des Toupouri en particulier. L'auteur analyse d'une part, les divers cheminements techniques que suivaient ce peuple pour exploiter le couvert végétal, et les multiples utilisations et transformations réservées aux arbres, d'autre part. $\mathrm{Au}$ total, ce sont les techniques utilisées dans l'architecture chez les Toupouri, et notamment celles relatives à la toiture des cases et aux greniers qui sont mises en exergue dans cette recherche.

L'étude conduite par la recherche que présente François Wassouni ${ }^{20}$, de son côté, analyse les techniques d'élaboration et d'usage du cuir au Nord-Cameroun. Il s'agit d'un matériau de grande importance, qui était transformé selon des techniques dont l'essentiel des intrants provenait de l'environnement. Cache-sexes qui tenaient lieu de vêtements, parures, matériel militaire, dispositifs techniques de la forge ou objets rituels, sont quelques-uns des objets fabriqués avec du cuir et qui avaient une grande place dans les sociétés du Nord-Cameroun précolonial, où les artisans avaient une technicité remarquable dans le domaine du cuir.

La problématique au centre de la réflexion de Noël Lavallière Betga Djenkwé21 est relative, pour sa part, aux techniques défensives des chefferies bamiléké au Cameroun, du XVIe au début du XXe siècle, un pan important de l'histoire militaire de ces sociétés. Elle pose le problème de l'ingéniosité qui a présidé à la protection des chefferies bamiléké contre les velléités des unes envers les autres, en inventoriant et analysant les réalisations techniques qui ont permis à ces différentes structures politiques 
d'assurer leur survie tout en préservant leurs biens et valeurs.

Par ailleurs, Mahamat Abba Ousman²2 aborde un sujet relatif au zemi. Il s'agit d'un outil de pêche ancien, en voie de disparition aujourd'hui aux abords du Lac Tchad, où des peuples comme les Kotoko, qui ont un lien tout particulier avec l'activité piscicole, l'utilisaient. Le zemi est étudié ici, tour à tour, à travers la technique et les moyens qu'il mobilisait, son usage, son impact socioéconomique sur l'aire culturelle kotoko et la dimension patrimoniale qu'il revêt. En filigrane, l'auteur propose des stratégies qui, mises en œuvre, pourraient permettre de sauvegarder et pérenniser cet élément technique aux enjeux mémoriels importants.

Enfin, le texte de Cyrille Zeh²3, qui clôt ce dossier, porte sur la filière vannerie au Cameroun. A travers l'analyse de ses techniques, de ses formes et de ses fonctions, ce travail montre que l'art de la vannerie utilise principalement comme ressources premières les rotins (filets, maraca) et d'autres matériaux tels que le bambou (Poaceae), le raphia (Arecaceae) et les lianes. Cette activité technique apparait comme une forme de création du patrimoine artistique local, qui ne s'inspire pas seulement des traditions artistiques des autres continents pour satisfaire la demande du monde cosmopolite de l'art et artisanat actuel, mais aussi des traditions artistiques africaines, pour mieux répondre aux besoin des sociétés africaines. C'est donc un secteur d'activité aux enjeux sociaux et économiques importants.

1 Titulaire d'un Doctorat/Ph.D. consacré à « L'artisanat du cuir dans l'Extrême-Nord du Cameroun du XIXe siècle à 2007 », François Wasssouni enseigne au département d'histoire de la Faculté des Lettres et Sciences Humaines de l'Université de Maroua (Cameroun). Professeur invité l'EHESS et à l'Université de Paris I Panthéon-Sorbonne (Master Erasmus Mundus TPTI), lauréat de plusieurs programmes de bourses internationales, auteur de plusieurs articles et co-directeur de deux ouvrages, il s’intéresse aux savoir-locaux africains, sous l'angle du patri- moine, des innovations techniques, et du tourisme en rapport avec le développement et la présence Chinoise en Afrique.

2 Revue Tiers Monde, $\mathrm{n}^{\circ}$ 216, octobre-décembre, Paris, Armand Colin/Dunod. Consultable sur : http://www.cairn.info/revuetiers-monde-2013-4-pages-7.htm

3 COQUERY-VIDROVITCH C., "L'historiographie africaine en Afrique », Revue Tiers Monde, $\mathrm{n}^{\circ}$ 216, octobre-décembre, Paris, Armand Colin/Dunod, pp. 111-127.

4 Entretien réalisé par Chloé MAUREL, "Comment une maison d'édition française donne la parole aux historiens des Suds», Revue Tiers Monde, $\mathrm{N}^{\circ}$ 216, octobre-décembre, Paris, Armand Colin/Dunod, pp. 129-140.

5 COQUERY-VIDROVITCH C., pp. 111-127.

6 On ne saurait dresser un catalogue des revues consacrées aux techniques. On peut citer entre autres, Documents pour l'histoire des techniques; e-Phaïstos; Artefact. Techniques, histoire et Sciences Humaines.

7 Le Centre de recherches en histoire des sciences et des techniques du Centre Alexandre Koyré; Centre d'histoire des sciences et d'histoire des techniques, composante de l'IHMC à l'Université Paris I Panthéon-Sorbonne; Master Erasmus Mundus Techniques, Patrimoine, Territoire de l'Industrie (TPTI) ; le laboratoire RECITS à l'Université de technologie BelfortMontbéliard; le laboratoire Histoire des technosciences en société (HT2S) logé au sein du Conservatoire nationale des Arts et Métiers (CNAM) de Paris; Centre François Viète de l'Université de Nantes, entre autres.

8 On peut citer Hamady BOCOUM, L’âge du fer au Sénégal: histoire et archéologie, Dakar, IFAN Ch. A. Diop, 2000; KIETHEGA J.B., La métallurgie lourde du fer au BurkinaFaso : une technologie à l'époque précoloniale, Paris, Karthala, 2009 ; KIETHEGA J.B., L'or de la Volta noire : archéologie et histoire de l'exploitation traditionnelle, région de Poura, HauteVolta, Paris, Karthala, 1983; LEBEUF J.P., Archéologie tchadienne ; (les Sao du Cameroun et du Tchad), Paris, Hermann, 1968 ; GAST M., SIGAUT F. et al. (dir.), Les techniques de conservation des grains à long terme. Leur rôle dans la dynamique des systèmes de culture et des sociétés, Paris, CNRS Éditions, 1979,1981 et 1985 ; THIERNO MOUCTAR Bah et GHOMSI E., "Problématique des transmissions des techniques à travers le Sahara du VIII e au XVIe siècles », Afrika Zamani, Revue d'histoire africaine, Yaoundé, février 1986, pp. 23- 35; MARZOUK Y., SEIGNOBOS Ch. Et SIGAUT F., (éds), Outils aratoires en Afrique. Innovations, normes et traces, Paris, Karthala-IRD, 2000 ; BARBOFF M., GRIFFIN-KREMER C. et SIGAUT F. (éds), Meules à grains, Paris, Ibis Press -Éditions de la MSH, 2003. 
9 Les travaux d'archéologues menés dans certaines parties du Cameroun et ceux des historiens comme ENGELBERT Mveng sur L'art et l'artisanat africains, Yaoundé, Clé, 1980, les mémoires et thèses sur les secteurs artisanaux, techniques militaires et de défense, constituent des références importantes.

$10 \mathrm{Du}$ développement des thèses racistes de HEGEL au XIX ${ }^{\mathrm{e}}$ siècle (La Raison dans l'histoire. Introduction à la philosophie de l'histoire (Die Vernunft in der Geschichte, (1822-1830), trad. UGE, 1965) au discours de Nicolas SARKOZY à Dakar (Sénégal) du 26 juillet 2007, de fausses et impertinentes idées ont été véhiculées sur l'incapacité des Africains à innover, à inventer.

11 DORTIER J. F. (dir.), "Techniques", Dictionnaire des Sciences Humaines, Évreux, Sciences Humaines Éditions, 2008, p. 708.

12 Introduction du cours d'A.-F.GARÇON sur « Histoire des objets techniques du XXe siècle », cours de Licence 2 de l'Université Paris 1 Panthéon-Sorbonne, consultable en ligne : https://sites.google.com/site/afgarcon/iht-introduction

13 DAUMAS, M. (éd.), Histoire générale des techniques, Vol. 1 : les origines de la civilisation technique, Paris, PUF, 1962.

14 Cours d'A-F. GARÇON, Op.cit.

15 Dans un article publié en 2012, je formulais un plaidoyer à l'endroit des historiens camerounais sur la nécessité et l'urgence de développer cette branche de l'histoire. Voir WASSOUNI F., "L'enseignement et la recherche en histoire des techniques à l'Université de Ngaoundéré au Cameroun entre 1993 et 2010 : bilan critique et perspectives ", e-Phaïstos, vol. I, ${ }^{\circ} 2$, décembre 2012, pp. 107-117.

16 GARÇON A.F., CARDOSO A. et LUIGI FONTANA G., L'aluminium et la calebasse. Patrimoines techniques, patrimoines de lindustrie en Afrique, Bialec (Nancy), Université de technologie de Belfort/Université de Paris I Panthéon-Sorbonne, 2013.

17 Il s'agit entre autres des noms comme Hamady BOCOUM, grand spécialiste du patrimoine métallurgique et acteur de la cause patrimoniale africaine, Jean-bapstiste Kiéthéga, fondateur de la paléométallurgie burkinabée et son collègue Lassina Koté.

18 Addo MAHAMANE est Maître de Conférences au Département d'histoire de l'Université Abdou Moumouni de Niamey. Actuellement Recteur de l'Université de Tahoua, il a publié un livre et plusieurs articles scientifiques sur des thèmes variés : le patrimoine culturel, l'Etat et l'armée, l'esclavage, entre autres. Il a été membre du Comité National chargé du suivi du Dossier de nomination de la ville d'Agadez au Patrimoine Mondial de l'UNESCO et du Comité Technique ad hoc pour la création du Musée des Forces Armées Nigériennes en 2010.
19 Jean GORMO enseigne au Département d'Histoire de la Faculté des Lettres et Sciences Humaines de l'Université de Maroua au Cameroun. Il s'intéresse depuis plusieurs années aux problématiques relatives à l'histoire environnementale. Il est auteur de deux livres: Maroua. Histoire du reboisement d'une ville sahélienne et Des arbres et des hommes. Une contribution à l'histoire des filières arboricoles à Maroua, Cameroun. Il est chercheur au Centre d'Etudes Africaines de l'Université de Porto (Portugal) et membre du Réseau Universitaire des Chercheurs en Histoire Environnementale (France).

20 Titulaire d'un Doctorat d'histoire soutenu à l'Université de Ngaoundéré au Cameroun sur "L'artisanat du cuir dans l'Extrême-Nord du Cameroun du XIXe siècle à 2007 », François WASSOUNI est enseignant au département d'histoire de la Faculté des Lettres et Sciences Humaines de l'Université de Maroua au Cameroun. Professeur Invité à l'Ecole des Hautes Etudes en Sciences Sociales (EHESS) de Paris en France, Visiting Scholar, Consortium du Master Erasmus Mundus Techniques, Patrimoine, Territoires de l'Industrie (TPTI), au Centre d'Etudes Africaines de Leiden aux Pays-Bas, lauréat de plusieurs programmes de bourses internationales, il a publié de nombreux articles sur l'histoire des techniques en Afrique. Il est le correspondant de la revue $e$-Phaïstos pour l'Afrique subsaharienne.

21 Noël Lavallière BETGA DJENKWE est depuis novembre 2011 Assistante au Département d'histoire à la Faculté des Arts, Lettres et Sciences Humaines de l'Université de Ngaoundéré. Elle est titulaire d'un Diplôme D'Etude Approfondie (DEA) en histoire, option Histoire politique et culturelle, obtenu à l'Université de Ngaoundéré en 2005. Elle est également détentrice d'un Master professionnel en Science politique, option Stratégie, Défense, Sécurité, Gestion des conflits et des catastrophes obtenu à l'Université de Yaoundé II en 2010. Le champ de recherche dans lequel elle mène depuis des recherches depuis sa maîtrise est celui de l'histoire militaire. Après le thème de la guerre depuis le XVIIe siècle dans l'Ouest-Cameroun, elle s'intéresse à la gestion post-conflit dans le cadre de sa thèse en préparation : « La (re)construction à l'Ouest-Cameroun (départements bamiléké) après la lutte pour l'indépendance du Cameroun sousadministration française (1962-1991) ».

22 Mahamat Abba OUSMAN est titulaire d'un doctorat en histoire culturelle et Chargé de Cours au Département des BeauxArts et des Sciences du Patrimoine de l'Institut Supérieur du Sahel, Université de Maroua au Cameroun. Il s'intéresse prioritairement au patrimoine culturel et à l'histoire des techniques. Il est auteur de plusieurs publications scientifiques, membre de la Société Camerounaise d'Histoire ( $\mathrm{SCH}$ ) et chercheur associé au Laboratoire de Conservation, Restauration et Recherches (LC2R) à Draguignan (France). 
23 Titulaire d'un CAP option Technologie des Arts Appliqués et d'un DEA en Histoire de l'Art, Cyrille ZEH est à la fois enseignant aux Département des Beaux Arts et Sciences du Patrimoine de l'Institut Supérieur du Sahel et plasticien. Il prépare actuellement une thèse de doctorat à l'Université de Maroua (Cameroun) portant sur la céramique ethnographique. Dans le cadre de sa formation, il explore des domaines et champs variés, notamment l'histoire de l'art (africain, contemporain, sacré), les arts visuels (céramique, sculpture, arts numériques et autres), la muséographie et le patrimoine. En dehors de nombreuses expositions artistiques (Yaoundé, Douala, Caire), il mène aussi des recherches dans les domaines du design et de l'esthétique industrielle. 